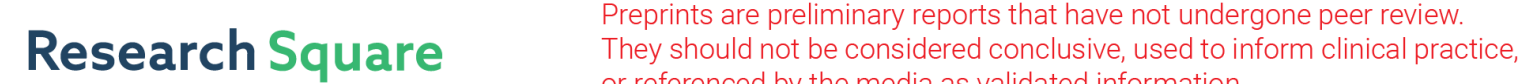 or referenced by the media as validated information.
}

\section{Determinants of Acceptance of Coronavirus Disease-2019 (COVID-19) Vaccine Among Lebanese Health Care Workers Using Health Belief Model}

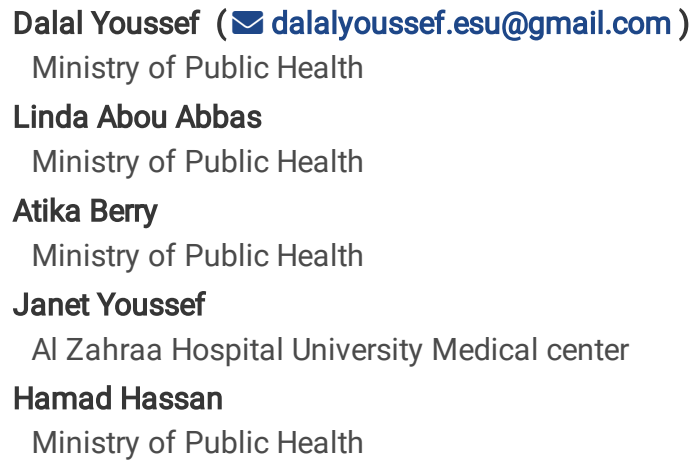




\section{Abstract}

Background: Since Health care workers (HCWs) are at high occupational risk for COVID-19, they are prioritized for immunization. This study aims to assess the acceptance rate of COVID-19 vaccine among HCWs and to identify its determinants.

Methodology: A web-based cross-sectional study was conducted over the period extending from 10 to 31 December 2020 among Lebanese HCWs. The Health Belief Model (HBM) was used as theoretical framework. Descriptive statistics were reported using frequency with percentages for categorical variables. Bivariate and multivariable logistic regression were carried out to identify the factors associated with the acceptance of COVID-19 vaccine. Adjusted odds ratio and their $95 \%$ confidence intervals were reported. The level of statistical significance was set at a $p$-value $<0.05$.

Results: A total of $1800 \mathrm{HCWs}$ have completed the survey. Around half of them were frontliners aged between (30-49) years old. About two third $(67.33 \%)$ have received their influenza vaccine this year. The acceptance rate of COVID-19 vaccine among surveyed HCWs was $58 \%$. Being a male (aOR=1.988, 95\% Cl (1.411-2.801)), working in the frontline (aOR=1.610,95\% $\mathrm{Cl}(1.173-2.209)$, and having received influenza vaccination this year $(\mathrm{aOR}=1.378,95 \% \mathrm{Cl}(0.990-1.916))$ were significantly associated with higher COVID-19 vaccine acceptance. However, living in rural area $(\mathrm{aOR}=0.606,95 \% \mathrm{Cl}(0.440-0.836))$, personal history of COVID-19 $(\mathrm{aOR}=0.695,95 \% \mathrm{Cl}(0.470-1.029)$, novelty of vaccine $(\mathrm{aOR}=0.424,95 \% \mathrm{Cl}$ (0.255-0.706)), side effects $(\mathrm{aOR}=0.413,95 \% \mathrm{Cl}(0.234-0.731)$, reliability of manufacturer (aOR=0.434, $95 \% \mathrm{Cl}(0.297-0.633)$ and vaccine frequency ( $\mathrm{aOR}=0.580,95 \% \mathrm{Cl}(0.403-0.836)$ were negatively associated with the willingness to take it. Remarkably, limited availability $(\mathrm{aOR}=2.161,95 \% \mathrm{Cl}(1.461-3.197)$ and accessibility $(\mathrm{aOR}=1.680,95 \% \mathrm{Cl}(1.141-2.474)$ to the vaccine were also positively associated to its uptake. Similarly, HCWs who perceived vaccine benefits were more likely to get vaccinated (aOR=11.048, 95\% $\mathrm{Cl}(7.993-15.269)$ ). With regards to cues of action, having reliable and adequate information regarding the vaccine (aOR=1.979, $95 \% \mathrm{Cl}(1.361-2.878)$ ), recommendation by health authorities $(\mathrm{aOR}=1.976,95 \% \mathrm{Cl}(1.343-2.908))$ or health facilities $(\mathrm{aOR}=2.684,95 \% \mathrm{Cl}(1.804-3.994))$ were positively linked to vaccine acceptance, whilst recommendation by family members ( $\mathrm{aOR}=0.479,95 \% \mathrm{Cl}(0.283-0.811))$ was negatively related to it.

\section{Conclusion:}

Pointing out the factors driving moderate vaccine acceptance among HCWs via targeting messages addressing key concerns would be required to reach higher vaccination rate.

\section{Background}

Coronavirus disease 2019 (COVID-19) has instigated a public health and economic calamity worldwide [1]. It continues to inflict substantial morbidity and mortality despite intervention efforts. As of 20 January 2021, there have been over 85 million cases and 1.8 million deaths reported [2]. To control the blowout of COVID-19, countries have focused their efforts on slowing the spread of the disease by adopting a variety of non-pharmaceutical interventions, including travel restriction, schools' closure, remote schooling, forced quarantine, and lockdown [3]. Despite that these measures are considered essential in the short-term, a long-lasting solution represented by vaccines is urgently needed to dramatically lessen the mortality burden and conceivably halt local transmission [4].

Considering this fact, numerous pharmaceutical companies were preemptive, as they took the initiative to started vaccine development since the early phase of the pandemic, which coincided with the publication of the first genome sequence of the Severe acute respiratory syndrome coronavirus 2 (SARS-CoV-2) [5]. Several prophylactic vaccines against COVID-19, including mRNA-based vaccine, DNA-based vaccines, inactivated, live attenuated, sub-unit, and replicating or no replicating viral vector-based vaccines are being developed across multiple countries [6]. Fortunately, the rapid development of many promising vaccines was crowned in the final weeks of 2020 by the first authorization and shipping of doses [7]. Correspondingly, many countries have authorized the emergency use of COVID-19 vaccines [8].

However, limited COVID-19 vaccine supply combined with the vibrant discrepancies in the transmission dynamics and seriousness of COVID-19 across groups will be extremely challenging for the first several months of the vaccination campaign[9]. Accordingly, it urges the need to establish an effective policy for COVID-19 vaccine allocation based on a deep understanding of the current epidemiological characteristics of COVID-19 [10]. This information includes key factors of group heterogeneity such as susceptibility to get COVID-19, the severity of outcomes, and contact rates. However, vaccination policy against influenza could not be used as a mirror for the ongoing COVID-19 pandemic. This can be due to the sharp variances in the epidemiology of the 2 viruses. For example, lower susceptibility to infection among children and adolescents and a substantial higher fatality rate that increases evidently with age is associated with COVID-19 [11].

Given the evidence of ongoing COVID-19 infections among HCWs and the high occupation risk encountered by them particularly those working in the frontline, the need to protect them is considered a national priority [12]. Besides, their role in providing care to patients and the negative impact resulting from their sickness if they got COVID-19 on medical services provided by health facilities, they can provoke nosocomial outbreak by spreading the virus to those they are caring for hospitalized patients and residents of health facilities [13]. 
Early vaccine access for HCWs is critical to ensuring their health and safety, thus protecting their patients, families, communities, and the broader health of our country. Based on the Values Framework and Population Prioritization Roadmap, issued by a World Health Organization (WHO) advisory group on immunization, countries that plan to roll out COVID-19 should prioritize health care workers (HCWs) and other at-risk populations for vaccination [14].

In Lebanon, since the early phase of vaccine development, national efforts were engrossed in securing the country's portion of the COVID-19 vaccine through negotiation conducted with Pfizer/BioNtech Company. The vaccine is intended to reach Lebanon by mid-February 2021 and the expectable supply will cover $15 \%$ of the population. The vaccine will be provided for free for the population following a voluntary process. In addition, the COVAX platform will cover $20 \%$ of the population [15]. As of March 2, 2021, there were more than 2567 confirmed COVID-19 cases and 45 deaths reported among HCWs in Lebanon [16]. Similarly, to other countries and based on the current epidemiological data, the MOPH has prioritized the COVID-19 vaccination for all HCWs. Hence, it is crucial that government start to gauge current levels of willingness to receive a potentially safe and effective COVID-19 vaccine among HCWs and to identify determinants of acceptance and refusal of the vaccine [17].

The objectives of this study were to assess the acceptance rate of the COVID-19 vaccine among HCWs and to evaluate the perceived susceptibility to COVID-19, perceived severity of COVID-19 infection, perceived benefits, barriers of COVID-19 vaccine, and appraise participants' cues to action and self-efficacy. In addition, we sought to identify the determinants of participants' intention to receive the COVID-19 vaccine.

\section{Methods}

\section{Study tool and design:}

A cross-sectional study, using an online survey, was conducted during the early phase preceding the arrival of the COVID-19 vaccine in Lebanon between 10th and 31st December 2020. As the Lebanese government recommended the public to minimalize face-to-face interaction, potential respondents were electronically invited to participate.

\section{Questionnaire development}

An extensive review of the literature was conducted to list available resources on acceptance of vaccine, as well as to identify relevant items and scales on vaccination. The Health Belief Model (HBM), used extensively in vaccination research to study vaccination behaviors and to identify participant perceptions towards disease and vaccination was adopted as a theoretical framework in this study in order to assess HCWs drives for receiving COVID-19 vaccine [18]. It comprises six key domains which impact willingness to vaccinate: perceived susceptibility to COVID-19, perceived severity of COVID-19 infection, perceived benefits of COVID-19 vaccine, perceived barriers of COVID-19 vaccine, cues to action, and self-efficacy [19].

A 72-item questionnaire was developed to assess the study objectives. A structured questionnaire was initially developed and designed by the authors to cover important aspects of COVID-19 vaccination among HCWs based upon the HBM (Supplement file 1).

A panel of experts has reviewed the developed questionnaire and assessed the clarity of the questions, interpretability, and accuracy of the domains. Then he original English draft of the questionnaire was translated and adapted to the Arabic language based on standard translation guidelines [20]. The questionnaire was pre-tested among $20 \mathrm{HCWs}$ for survey flow, functionality, readability, comprehension of instructions, and clarity. Based upon feedback from the pre-test, minor modifications regarding readability and clarity were made to the questionnaire. Furthermore, the reliability of the questionnaire was checked, and the Cronbach Alpha value was 0.82 . The average time for filling the survey was 8 minutes. The questionnaire was self-administered and consisted of open-ended questions. It was divided into seven domains:

1. Baseline information of participants: including age, gender, marital status, urbanicity, and specialty, place of work, clinical experience, health status, underlying conditions, household size, household income, religiosity, and health coverage. Participants were also asked whether they have a child at home or they live with an elderly or someone having comorbidities. Besides, they were asked if they have ever contracted COVID-19 or they have one family member or colleague previously infected with COVID-19. They were also asked if they have received the influenza vaccine and if they have refused any kind of vaccination previously.

2. The perceived susceptibility to COVID-19 section. It consists of 4 questions addressing HCW's views about their risk of getting COVID-19 [21].

3. The perceived severity section consists of 5 questions that relate to the patient's concerns about the seriousness of COVID-19.

4. The perceived benefits section comprises 7 questions linked to the perceived outcomes of getting vaccinated against COVID-19 to reduce their susceptibility to or severity of the illness [22].

5. Perceived barriers consist of 13 questions that identify patient's concerns or negative beliefs about COVID-19 vaccines. Responses to questions related to perceived susceptibility, severity, benefits, and barriers were graded on a 3-point Likert scale, an agreement scale ranging from ' 1 ' for disagree to ' 3 ' agree. 
6. The cues to action section comprise 7 questions addressing strategies or information sources that promote the willingness to get the COVID-19 vaccine. Responses to statements related to cues of action were sorted on a 3-point Likert scale, an agreement scale ranging from ' 1 ' for No, '2' Not sure and '3' Yes.

7. The self-motivation section comprised 2 statements that addressed HCW's willingness to improve his health, e.g., adopting a healthy lifestyle. Responses to questions related to self-motivation were graded on a 3-point Likert scale, an agreement scale ranging from ' 1 ' for never to ' 3 ' all the time[23].

8. Knowledge about vaccine section consists of 8 questions assessing awareness of HCWs about COVID-19 vaccine. All the items were answered on a true/false basis and an additional "do not know" option. A correct response had a value of ' 1 ' and a "wrong" or don't know response had a value of ' 0 '. Hence, the aggregate score for all 8 knowledge questions would range from 0 to 8 points. Participants 'overall knowledge was categorized using modified Bloom's cut-off point, as good if the score was $>60 \%$ ( $5-8$ points), and poor if the score was less than $60 \%$ (< 5 points)

Participants were also requested to indicate their intention to receive the COVID-19 vaccine and to rank the reliability of information sources.

\section{Sample size calculation}

To calculate the sample size of the study, the Raosoft sample size calculator designed specifically for population surveys was used. Assuming that between 50000 registered HCWs, 40000 of them are actively practicing at the health facilities level, a $95 \%$ confidence level was used and an absolute error was estimated to be $5 \%$. All previous information was used to calculate the sample size for this study which yielded the least required sample size of 381 participants. The required sample size was achieved at an early stage before the closure of response acceptance (January $\left.1^{\text {st }}, 2021\right)$.

\section{Data collection}

An online questionnaire using a Google form was emailed to governmental run and private hospital directors. Then, designated focal persons working in Lebanese hospitals were contacted via phone call and notified about the survey and its purpose. Upon their agreement to participate, the link of the study was sent through "WhatsApp" to the designated focal person (infection control personnel) who was requested in his turn to disseminate it among other HWCs facilities. This link included a brief introduction to the background, the objective of the survey, and instructions for filling the questionnaire.

HCW is defined as: any regulated health professionals and any staff member, or other essential caregiver currently working in a health care organization, including cleaning staff, food services staff, and other administrative staff [24]. Participants were identified via the infection control personnel at the hospital. All HWCs, working in Lebanese hospitals in different provinces in Lebanon and who agreed to participate to the study, were eligible for participation.

\section{Ethical considerations}

An oral informed consent was obtained for each participant. They were reassured that their participation is voluntary and that they were free to withdraw at any time. In addition, all information were gathered anonymously and handled confidentially. The questionnaire was collected only in subjects who expressed consent for study participation. As individual participants cannot be identified based on the presented material, this study caused no plausible harm or stigma to participants. The study design assured adequate protection of study participants, and neither included clinical data about patients nor configured itself as a clinical trial. Hence, this study was exempted from ethical approval of the ministry of Public Health.

\section{Statistical analysis}

The data were analyzed using the statistical software SPSS (Statistical Package for Social Sciences), version 22.0. Descriptive statistics were reported using frequency with percentages for categorical variables. Bivariate analysis was performed to examine factors associated with the dependent variable (willingness to vaccinate) and the demographic variables in addition to the domains of HBM. The relation between nominal variables was tested using the chi-squared test. The variables in bivariate analysis with $\mathrm{p}$-value $<0.2$ were entered into multivariable logistic regression. Adjusted odds ratio and their $95 \%$ confidence intervals were reported. The final logistic regression model to determine the predictors of willingness to vaccinate was reached after confirming the adequacy of the data using the Hosmer and Lemeshow test. The level of statistical significance was set at a p-value $<0.05$.

\section{Results}

A total of $1800 \mathrm{HCW}$ completed the survey. The majority of participants were female $1209(67.1 \%)$ and around half of them were aged between (30-49) years old (Table 1). About two third of the HCWs were married (62.6\%) and reside in urban area (61.4\%). The higher 
participation rate was among nurses (48.9\%). They mostly had a good health status (81.4\%) and were covered by a public insurance (79.60\%). Only $22 \%$ of HCWs suffered from comorbidities. Approximately all the participants (91.9\%) reported having a colleague infected by COVID-19 and only $20.9 \%$ of them reported being infected by COVID-19. More than half of the participants (58.10\%) were frontliners in the response to COVID-19.

Only $45.4 \%$ of surveyed HCWs have received influenza vaccine in the past season whereas $67.33 \%$ of them declared that they got vaccinated against influenza during the current season. Only $18.94 \%$ of them had reported that they refused a type of vaccine other than influenza in the past. However, only $58 \%$ of surveyed HCWs said that they would get vaccinated if the COVID-19 vaccine was available (figure 1 ). Figure 2 showed that the intention of HCWs to receive COVID-19 vaccine increased with age. Variance in acceptance rate of COVID-19 vaccine was identified also between geographical areas where Akkar reported the lowest rate (42.6\%), compared to Beirut (Capital) that ranked the top (Figure 3).

Of the total, $86.5 \%$ of participants perceived themselves susceptible to get COVID-19 due to their occupational exposure and $91.6 \%$ of them considered that healthy people can get COVID-19. Only half of them declared that they can protect themselves better than other people. More than $90 \%$ of HCWs agreed that COVID-19 makes some people ill and could be fatal and $85.6 \%$ considered that COVID-19 is more serious than influenza. Only $29.3 \%$ of them thought that they will be very ill if they contracted COVID-19, $30.7 \%$ stated that they may need hospitalization and $21.7 \%$ pondered that they might die (Table 2 ).

Table 3 displayed perceived benefits and barriers of COVID-19 vaccination by HCWs. Around half of them agreed that vaccination is a good idea because it makes them feeling less worried about catching COVID-19, it decreases their chance of getting COVID-19 or its complications. They reported that if they got vaccinated, they could protect their close environment (patients, family and friends) from the infection and the whole community in wider range. The major perceived barriers cited by HCWs for COVID-19 vaccine were the novelty, the side effects, the efficacy and the safety of the vaccine.

Table 4 summarized cues of action that promote willingness to get COVID-19 vaccine. The majority of HCWs (64.3\%) stated that they will take the COVID-19 vaccine if they had reliable information about it and $47.7 \%$ of them will took the vaccine if it is recommended by the health authorities. Third quarter of respondents said that they frequently did things on their own to improve their health (healthy diet, physical diet...) and followed yearly physical examinations in addition to visits related to illness.

Table 5 displayed the HCWs responses to the knowledge items related to the vaccine. Approximately two quarters of them (76.8\%) were found to be knowledgeable about the basic information related to COVID-19 vaccines (Figure 4). 


\begin{tabular}{|c|c|c|}
\hline & $\mathrm{n}$ & $\%$ \\
\hline \multicolumn{3}{|l|}{ Gender } \\
\hline Male & 593 & $32.90 \%$ \\
\hline Female & 1209 & $67.10 \%$ \\
\hline \multicolumn{3}{|l|}{ Age } \\
\hline $18-29$ years & 651 & $36.20 \%$ \\
\hline $30-49$ years & 904 & $50.20 \%$ \\
\hline$>50$ years & 245 & $13.60 \%$ \\
\hline \multicolumn{3}{|l|}{ Marital status } \\
\hline Single & 618 & $34.30 \%$ \\
\hline Married/Engaged & 1127 & $62.60 \%$ \\
\hline Other (Divorced or Widowed) & 55 & $3.10 \%$ \\
\hline \multicolumn{3}{|l|}{ Urbanicity } \\
\hline Rural & 695 & $38.60 \%$ \\
\hline Urban & 1105 & $61.40 \%$ \\
\hline \multicolumn{3}{|l|}{ Occupation } \\
\hline Physician & 382 & $21.20 \%$ \\
\hline Nurse & 880 & $48.90 \%$ \\
\hline Pharmacist & 124 & $6.90 \%$ \\
\hline Administrative & 206 & $11.40 \%$ \\
\hline Others (Midwife, Lab technician...) & 208 & $11.6 \%$ \\
\hline \multicolumn{3}{|l|}{ Health status } \\
\hline Fair and Below & 335 & $18.60 \%$ \\
\hline Good and above & 1465 & $81.40 \%$ \\
\hline \multicolumn{3}{|c|}{ Presence of underlying health condition } \\
\hline Yes & 396 & $22.00 \%$ \\
\hline No & 1404 & $78.00 \%$ \\
\hline \multicolumn{3}{|l|}{ Presence of child at home } \\
\hline Yes & 1000 & $55.60 \%$ \\
\hline No & 800 & $44.40 \%$ \\
\hline \multicolumn{3}{|l|}{ Presence of elderly people at home } \\
\hline Yes & 852 & $47.30 \%$ \\
\hline No & 948 & $52.70 \%$ \\
\hline \multicolumn{3}{|l|}{ Health insurance } \\
\hline Public & 1433 & $79.60 \%$ \\
\hline Private & 220 & $12.20 \%$ \\
\hline None & 147 & $8.20 \%$ \\
\hline \multicolumn{3}{|c|}{ Working in frontline in the response to COVID-19 } \\
\hline Yes & 1045 & $58.10 \%$ \\
\hline
\end{tabular}

Page 6/19 


\begin{tabular}{|c|c|c|}
\hline No & 755 & $41.90 \%$ \\
\hline \multicolumn{3}{|c|}{ Personal history of COVID-19 diagnosis } \\
\hline Yes & 377 & $20.90 \%$ \\
\hline No & 1423 & $79.10 \%$ \\
\hline \multicolumn{3}{|c|}{ Family member/friend ever diagnosed with COVID-19 } \\
\hline Yes & 713 & $39.60 \%$ \\
\hline No & 1087 & $60.40 \%$ \\
\hline \multicolumn{3}{|c|}{ Colleague ever diagnosed with COVID-19 } \\
\hline Yes & 1654 & $91.90 \%$ \\
\hline No & 146 & $8.10 \%$ \\
\hline Total & 1800 & $100 \%$ \\
\hline
\end{tabular}

Figure 5 displayed the reliability of sources of information used by HCWs. International Health Websites (WHO...) and scientific articles were ranked the most reliable sources, while family and friends were listed the least trusted information sources.

\section{Logistic Regression:}

Table 6 presents the multivariable logistic regression of the factors associated with COVID-19 vaccine acceptance. Our results showed that being a male $(\mathrm{aOR}=1.988,95 \% \mathrm{Cl}(1.411-2.801)$ ), working in the frontline (aOR=1.610,95\% $\mathrm{Cl}(1.173-2.209)$, and having received influenza vaccination in the current season (aOR=1.378, 95\% $\mathrm{Cl}(0.990-1.916))$ were significantly associated with higher COVID-19 vaccine acceptance. However, living in rural area $(\mathrm{aOR}=0.606,95 \% \mathrm{Cl}(0.440-0.836))$ and having personal history of COVID-19 (aOR=0.695, 95\% Cl (0.470-1.029) were negatively associated to the COVID-19 vaccine uptake. Similarly, the vaccine acceptance was lower in HCWs who perceived barriers such us novelty of vaccine $(\mathrm{aOR}=0.424,95 \% \mathrm{Cl}(0.255-0.706))$, side effects $(\mathrm{aOR}=0.413,95 \% \mathrm{Cl}(0.234-0.731)$, reliability of manufacturer $(\mathrm{aOR}=0.434$, $95 \% \mathrm{Cl}(0.297-0.633)$ and vaccine frequency $(\mathrm{aOR}=0.580,95 \% \mathrm{Cl}(0.403-0.836)$. Remarkably, limited availability (aOR=2.161, 95\% Cl(1.461-

3.197) and accessibility (aOR=1.680, 95\% $\mathrm{Cl}(1.141-2.474)$ to the vaccine were positively associated with high vaccine uptake. Likewise, $\mathrm{HCWs}$ who perceived vaccine benefits were 11 times more likely to get vaccinated (aOR=11.048, 95\% $\mathrm{Cl}(7.993-15.269)$ ) than their counterparts, it is the main driver of vaccine uptake. With regards to cues of action, having reliable and adequate information regarding the vaccine (aOR=1.979, $95 \% \mathrm{Cl}(1.361-2.878))$, recommendation by health authorities (aOR=1.976, 95\% $\mathrm{Cl}(1.343-2.908)$ ) or health facilities (aOR=2.684, 95\% $\mathrm{Cl}(1.804-$ $3.994)$ ) were positively linked to vaccine acceptance, whilst recommendation by family members $(\mathrm{aOR}=0.479,95 \% \mathrm{Cl}(0.283-0.811))$ was negatively related to it. 


\begin{tabular}{|c|c|c|c|c|}
\hline & & Disagree & Neutral & Agree \\
\hline & & $\mathrm{n}(\%)$ & $\mathrm{n}(\%)$ & $\mathrm{n}(\%)$ \\
\hline & \multicolumn{4}{|l|}{ Perceived susceptibility to COVID-19 } \\
\hline S1 & I am susceptible of getting infected due to my occupational exposure & $46(2.6 \%)$ & 197(10.9\%) & $1557(86.5 \%)$ \\
\hline S2 & $\begin{array}{l}\text { There is a great chance to get infected by COVID-19 in the next coming months } \\
\text { especially during winter }\end{array}$ & $119(6.6 \%)$ & $430(23.9 \%)$ & $1251(69.5 \%)$ \\
\hline S3 & Healthy people can get COVID-19 & $47(2.6 \%)$ & $104(5.8 \%)$ & $1649(91.6 \%)$ \\
\hline S4 & My health status makes me more susceptible to contract COVID-19 & $897(49.8 \%)$ & $526(29.2 \%)$ & $377(20.9 \%)$ \\
\hline \multirow[t]{2}{*}{ S4 } & I believe that I can protect myself against COVID-19 better than other people & $242(13.4 \%)$ & $614(34.1 \%)$ & $944(52.4 \%)$ \\
\hline & \multicolumn{4}{|l|}{ Perceived severity and seriousness } \\
\hline Sev1 & $\begin{array}{l}\text { Although for most people, COVID-19 causes mild illness, it makes some people very } \\
\text { ill and can be fatal }\end{array}$ & $24(1.3 \%)$ & $140(7.8 \%)$ & $1636(90.9 \%)$ \\
\hline Sev2 & I think COVID-19 is more serious than seasonal influenza & $81(4.5 \%)$ & $178(9.9 \%)$ & $1541(85.6 \%)$ \\
\hline Sev3 & I will be very sick if I get COVID-19 & $345(19.2 \%)$ & $927(51.5 \%)$ & $528(29.3 \%)$ \\
\hline Sev4 & If I get COVID-19, I might require hospitalization & $415(23.1 \%)$ & $832(46.2 \%)$ & $553(30.7 \%)$ \\
\hline Sev5 & If I get COVID-19, I might die & $520(28.9 \%)$ & $890(49.4 \%)$ & $390(21.7 \%)$ \\
\hline
\end{tabular}


Table 3: HCW's perception of benefits and barriers of COVID-19

\begin{tabular}{|c|c|c|c|c|}
\hline & & Disagree & Neutral & Agree \\
\hline & & $\mathrm{n}(\%)$ & $\mathrm{n}(\%)$ & $\mathrm{n}(\%)$ \\
\hline & \multicolumn{4}{|l|}{ Perceived benefits } \\
\hline 11 & $\begin{array}{l}\text { Vaccination is a good idea because it makes me feel less worried about catching } \\
\text { COVID-19 }\end{array}$ & $421(23.4 \%)$ & $464(25.8 \%)$ & $915(50.8 \%)$ \\
\hline 12 & Vaccination decreases my chance of getting COVID-19 or its complications & $326(18.1 \%)$ & $513(28.5 \%)$ & $961(53.4 \%)$ \\
\hline 13 & Vaccines are considered between the most tested and safe medical products & $327(18.2 \%)$ & $658(36.6 \%)$ & $815(45.35)$ \\
\hline 14 & When I get vaccinated, I protect my patients, family and friends from infection & $330(18.3 \%)$ & $540(30 \%)$ & $930(51.7 \%)$ \\
\hline 15 & $\begin{array}{l}\text { When I get vaccinated, the whole community benefits by preventing the spread of } \\
\text { COVID-19 }\end{array}$ & $285(15.8 \%)$ & $536(29.8 \%)$ & $979(54.4 \%)$ \\
\hline 16 & COVID-19 vaccination is an effective way to prevent and control COVID-19 & $251(13.9 \%)$ & $541(30.1 \%)$ & $990(55 \%)$ \\
\hline \multirow[t]{2}{*}{17} & High vaccination coverage globally is required to stop COVID-19 pandemic & $225(12.5 \%)$ & $571(31.7 \%)$ & $1004(55.8 \%)$ \\
\hline & Perceived barriers & & & \\
\hline B1 & I am concerned about the novelty of vaccine (not used before) & $151(8.4 \%)$ & $386(21.4 \%)$ & $1263(71.1 \%)$ \\
\hline B2 & I am concerned about the side effects of COVID-19 vaccine & $134(7.4 \%)$ & $330(18.3 \%)$ & $1336(74.2 \%)$ \\
\hline B3 & I am concerned about the efficacy of COVID-19 vaccine & 193(10.7\%) & $342(19 \%)$ & $1265(70.3 \%)$ \\
\hline B4 & I am concerned about the safety of COVID-19 vaccine & $156(8.7 \%)$ & $371(20.6 \%)$ & $1273(70.7 \%)$ \\
\hline B5 & I am concerned about the cost of COVID-19 vaccine (willingness to pay) & $429(23.8 \%)$ & $539(29.9 \%)$ & $832(46.2 \%)$ \\
\hline B6 & $\begin{array}{l}\text { I am concerned about the accessibility of COVID-19 vaccines (geographical } \\
\text { distribution of sites) }\end{array}$ & $529(29.6 \%)$ & $563(31.3 \%)$ & $708(39.3 \%)$ \\
\hline B7 & $\begin{array}{l}\text { I am concerned about the availability of COVID-19 vaccine in limited quantities for } \\
\text { limited categories of the population }\end{array}$ & 239(13.3\%) & $475(26.4 \%)$ & $1086(60.3 \%)$ \\
\hline B8 & I am concerned whether if the COVID-19 vaccine is halal & $862(47.9 \%)$ & $546(30.3 \%)$ & $392(21.8 \%)$ \\
\hline B9 & I am concerned about the reliability of the manufacturer and the source of supply & $329(18.3 \%)$ & $573(31.8 \%)$ & $898(49.9 \%)$ \\
\hline B10 & $\begin{array}{l}\text { I am concerned about Lebanese health system, and the strategy of distribution of the } \\
\text { vaccines }\end{array}$ & $157(8.7 \%)$ & $452(25.1 \%)$ & $1191(66.2 \%)$ \\
\hline B11 & I am concerned about vaccine mode of administration (needles use...) & $709(39.4 \%)$ & $518(28.8 \%)$ & $573(31.8 \%)$ \\
\hline B12 & I am concerned about vaccine frequency (number of doses required....) & $510(28.3 \%)$ & $559(31.1 \%)$ & $731(40.6 \%)$ \\
\hline B13 & I am concerned about immunity duration (how much time I will be protected) & $156(8.7 \%)$ & $493(27.4 \%)$ & $1151(63.9 \%)$ \\
\hline
\end{tabular}




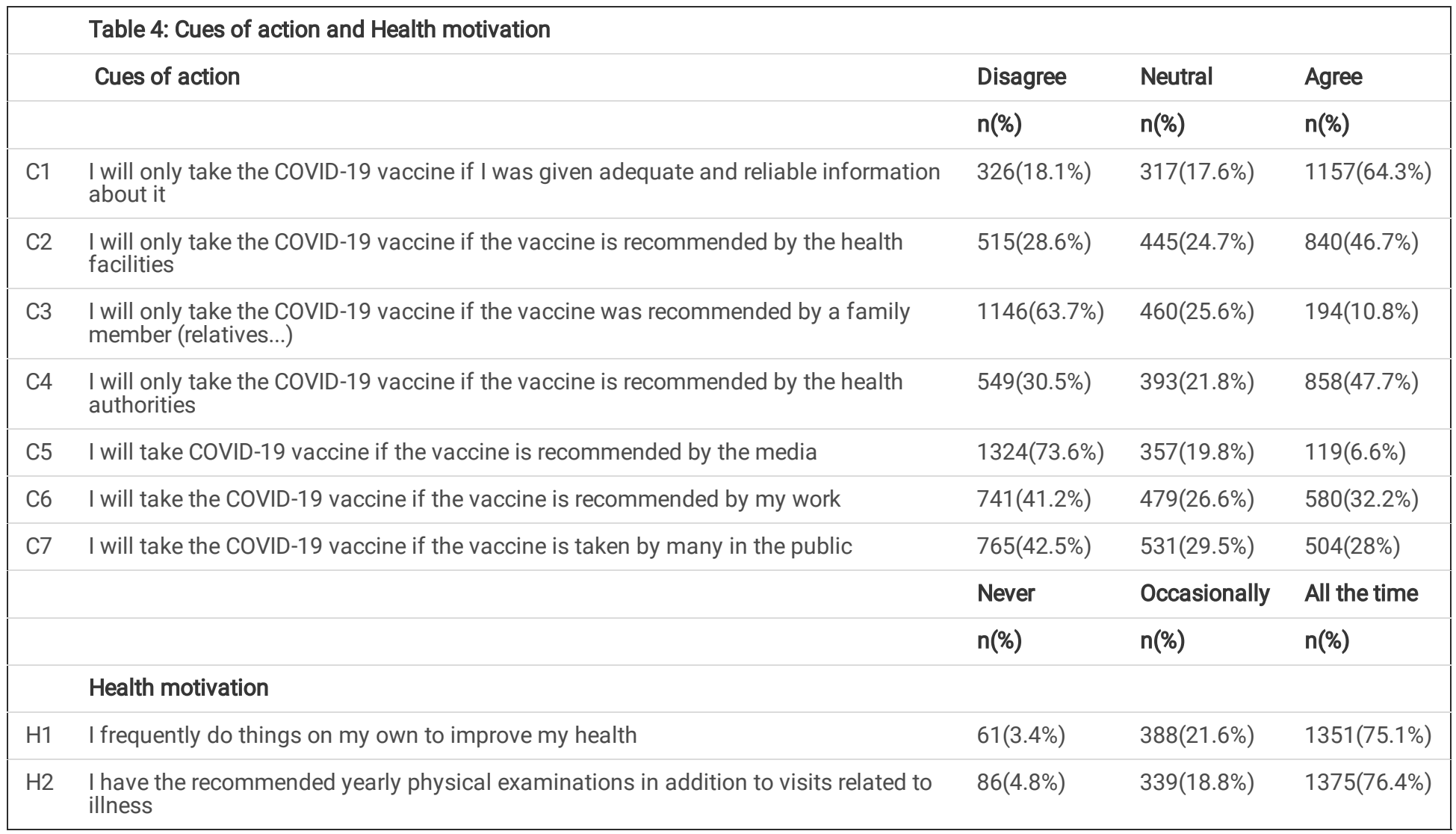

Table 5: HCWs' responses Knowledge items

\begin{tabular}{|c|c|c|c|c|}
\hline & & Correct & Incorrect & $\begin{array}{l}\text { I Don't } \\
\text { Know }\end{array}$ \\
\hline & & $n(\%)$ & $n(\%)$ & $\mathrm{n}(\%)$ \\
\hline \multicolumn{5}{|c|}{ Knowledge about vaccine } \\
\hline K1 & Vaccines are effective in combating highly contagious diseases ${ }^{c}$ & $1307(72.6 \%)$ & $97(5.4 \%)$ & $396(22 \%)$ \\
\hline K2 & $\begin{array}{l}\text { Traditionally, vaccines create immunity by introducing a weak form of an infectious } \\
\text { agent that allows the immune system to build a memory against this agent }{ }^{c}\end{array}$ & $1358(75.4 \%)$ & $44(2.4 \%)$ & $398(22.1 \%)$ \\
\hline K3 & $\begin{array}{l}\text { The RNA and DNA vaccines give our bodies the genetic code it needs to allow our } \\
\text { immune system to produce the antigen on its own }{ }^{c}\end{array}$ & $972(54 \%)$ & $83(4.6 \%)$ & $745(41.4 \%)$ \\
\hline K4 & $\begin{array}{l}\text { Covid-19 vaccines are being developed as quickly as possible, but they were required } \\
\text { receive the necessary regulatory licenses }{ }^{c}\end{array}$ & $740(41.1 \%)$ & $157(8.7 \%)$ & $903(50.2 \%)$ \\
\hline K5 & The flu vaccine protects against covid- $19^{\mathrm{F}}$ & $1283(71.3 \%)$ & $78(4.3 \%)$ & $439(24.4 \%)$ \\
\hline K6 & $\begin{array}{l}\text { People with chronic diseases and elderly are more likely to have the disease and its } \\
\text { complications, so they should get the vaccine }{ }^{c}\end{array}$ & $1334(74.1 \%)$ & $135(7.5 \%)$ & $331(18.4 \%)$ \\
\hline K7 & $\begin{array}{l}\text { Young people are healthy and therefore do not need to follow preventive measures and } \\
\text { to get the vaccine in order to protect themselves against Covid }-19^{\mathrm{F}}\end{array}$ & $1447(80.4 \%)$ & $138(7.7 \%)$ & $215(11.9 \%)$ \\
\hline K8 & $\begin{array}{l}\text { Until the readiness and the availability of COVID-19 vaccine, we cannot do anything to } \\
\text { tackle the disease } \mathrm{F}\end{array}$ & $1185(65.8 \%)$ & $331(18.4 \%)$ & $270(15 \%)$ \\
\hline
\end{tabular}




\begin{tabular}{|c|c|c|c|c|c|c|}
\hline & \multicolumn{6}{|c|}{ Acceptance of vaccine } \\
\hline & \multirow{2}{*}{$\begin{array}{l}\text { Yes } \\
\text { n (\%) }\end{array}$} & \multirow[t]{2}{*}{ P-value } & \multirow[t]{2}{*}{$\mathrm{aOR}$} & \multicolumn{2}{|c|}{$95 \% \mathrm{Cl}$ for aOR } & \\
\hline & & & & Lower & Upper & \\
\hline Gender & \multicolumn{6}{|c|}{$<0.001$} \\
\hline Female & $588(48.7 \%)$ & $619(51.3 \%)$ & & \multicolumn{3}{|l|}{1.00} \\
\hline Male & $161(27.2 \%)$ & $432(72.8 \%)$ & & 1.988 & 1.411 & 2.801 \\
\hline Urbanicity & \multicolumn{6}{|c|}{0.002} \\
\hline Urban & $451(40.8 \%)$ & $654(59.2 \%)$ & & \multicolumn{3}{|l|}{1.00} \\
\hline Rural & $298(42.9 \%)$ & $397(51.7 \%)$ & & 0.606 & 0.440 & 0.836 \\
\hline Working in frontline in the response to COVID-19 & \multicolumn{6}{|c|}{0.003} \\
\hline No & $393(37.6 \%)$ & $652(62.4 \%)$ & & \multicolumn{3}{|l|}{1.00} \\
\hline Yes & $356(47.2 \%)$ & $399(52.8 \%)$ & & 1.610 & 1.173 & 2.209 \\
\hline Personal history of COVID-19 infection & \multicolumn{6}{|c|}{0.032} \\
\hline No & $571(40.1 \%)$ & $852(59.9 \%)$ & & \multicolumn{3}{|l|}{1.00} \\
\hline Yes & $178(47.2 \%)$ & $199(52.8 \%)$ & & 0.659 & 0.450 & 0.965 \\
\hline Received vaccination against influenza in the current season & \multicolumn{6}{|c|}{0.047} \\
\hline No & $296(50.3 \%)$ & $292(49.7 \%)$ & & \multicolumn{3}{|l|}{1.00} \\
\hline Yes & $453(37.4 \%)$ & $759(62.6 \%)$ & & 1.378 & 0.990 & 1.916 \\
\hline Refusal of any type of vaccination in the past & \multicolumn{6}{|c|}{0.039} \\
\hline No & $222(65.1 \%)$ & $119(34.9 \%)$ & & \multicolumn{3}{|l|}{1.00} \\
\hline Yes & $527(36.1 \%)$ & $932(63.9 \%)$ & & 0.695 & 0.470 & 1.029 \\
\hline Novelty of the vaccine (not used before) & \multicolumn{6}{|c|}{0.001} \\
\hline Disagree & $81(15.2 \%)$ & $453(84.8 \%)$ & & \multicolumn{3}{|l|}{1.00} \\
\hline Agree & $668(52.8 \%)$ & $598(47.2 \%)$ & & 0.424 & 0.255 & 0.706 \\
\hline Side effects of COVID-19 vaccine & \multicolumn{6}{|c|}{0.002} \\
\hline Disagree & $64(13.8 \%)$ & $400(86.2 \%)$ & & \multicolumn{3}{|l|}{1.00} \\
\hline Agree & $685(51.3 \%)$ & $651(48.7 \%)$ & & 0.413 & 0.234 & 0.731 \\
\hline Accessibility of COVID-19 vaccines (distribution of sites) & \multicolumn{6}{|c|}{0.009} \\
\hline Disagree & $566(51.8 \%)$ & $526(48.2 \%)$ & & \multicolumn{3}{|l|}{1.00} \\
\hline Agree & $183(25.8 \%)$ & $525(74.2 \%)$ & & 1.680 & 1.141 & 2.474 \\
\hline Availability of COVID-19 vaccine in limited quantities & \multicolumn{6}{|c|}{$<0.001$} \\
\hline Disagree & $455(63.7 \%)$ & $259(36.3 \%)$ & & \multicolumn{3}{|l|}{1.00} \\
\hline Agree & $294(27.1 \%)$ & $792(72.9 \%)$ & & 2.161 & 1.461 & 3.197 \\
\hline Reliability of the manufacturer and the source of supply & & & $<0.001$ & & & \\
\hline Disagree & $253(28 \%)$ & $649(72 \%)$ & & 1.00 & & \\
\hline Agree & $496(55.2 \%)$ & $402(44.8 \%)$ & & 0.434 & 0.297 & 0.633 \\
\hline Vaccine frequency (number of doses needed...) & & & 0.003 & & & \\
\hline Disagree & $383(35.8 \%)$ & $686(64.2 \%)$ & & 1.00 & & \\
\hline Agree & $366(50.1 \%)$ & $365(49.9 \%)$ & & 0.580 & 0.403 & 0.836 \\
\hline
\end{tabular}




\begin{tabular}{|c|c|c|c|c|c|c|}
\hline $\begin{array}{l}\text { I will take the COVID-19 vaccine if } \mathrm{i} \text { was given adequate and reliable } \\
\text { information about it }\end{array}$ & & & $<0.001$ & & & \\
\hline Disagree & $419(65.2 \%)$ & $224(34.8 \%)$ & & 1.00 & & \\
\hline Agree & $330(28.5 \%)$ & $827(71.5 \%)$ & & 1.979 & 1.361 & 2.878 \\
\hline $\begin{array}{l}\text { I will take the COVID-19 vaccine if the vaccine is recommended by the } \\
\text { health facilities }\end{array}$ & & & $<0.001$ & & & \\
\hline Disagree & $581(60.5 \%)$ & $379(39.5 \%)$ & & 1.00 & & \\
\hline Agree & $168(20 \%)$ & $672(80 \%)$ & & 2.684 & 1.804 & 3.994 \\
\hline $\begin{array}{l}\text { I will take the COVID- } 19 \text { vaccine if the vaccine was recommended by } \\
\text { a family member (relatives...) }\end{array}$ & & & 0.006 & & & \\
\hline Disagree & $699(43.5 \%)$ & $907(56.5 \%)$ & & 1.00 & & \\
\hline Agree & $50(25.8 \%)$ & $144(74.2 \%)$ & & 0.479 & 0.283 & 0.811 \\
\hline $\begin{array}{l}\text { I will take the COVID-19 vaccine if the vaccine is recommended by the } \\
\text { health authorities }\end{array}$ & & & $<0.001$ & & & \\
\hline Disagree & $596(63.3 \%)$ & $346(36.9 \%)$ & & 1.00 & & \\
\hline Agree & $153(17.8 \%)$ & $705(82.2 \%)$ & & 1.935 & 1.331 & 2.813 \\
\hline Perceived benefits scale & & & $<0.001$ & & & \\
\hline Non beneficial & $646(77.6 \%)$ & $186(22.4 \%)$ & & 1.00 & & \\
\hline Beneficial & $98(10.3 \%)$ & $852(89.7 \%)$ & & 11.048 & 7.993 & 15.269 \\
\hline
\end{tabular}

\section{Discussion}

As the COVID-19 continues to swiftly evolve into a full-blown pandemic, Lebanon is planning to initiate the first round of immunization within the first quarter of this year, slated HCWs and the elderly to be an early recipient of the COVID-19 vaccine. Since HCWs are at high occupational risk for COVID-19 and will be responsible to administer and recommend vaccines to their patients and the general population, hence the importance of gauging their willingness to get vaccinated and to identify the determinants of their COVID-19 vaccine acceptance. Besides, it allows us to proactively develop the appropriate interventions, particularly during the current flood of misinformation about the safety of the vaccine. To the best of our knowledge, this is the first large national study investigating both determinants of COVID-19 vaccine uptake among HCWs as well as their willingness to get the vaccine.

Our findings showed that $20.9 \%$ of surveyed HCWs reported a personal history of COVID-19 infection and $91.9 \%$ of them had a colleague infected by COVID-19. However, this infectiousness rate among Lebanese HCWs was higher than the reported rate in Qatar, where a study conducted among $16,912 \mathrm{HCW}$ revealed that $10.6 \%$ of HCWs were positive for COVID-19 and an exposure to a colleague (45\%) or a patient was stated [25]. In India, a study reported an 11\% prevalence of COVID-19 infection among HCWs [26]. This could be due to the lack of sufficient personal protective equipment (PPEs) at facilities, their proper use and poor adherence to other infection control measures. Therefore, our results underline the need of effective HCWs protection.

More than half of our participants were working in the frontline of the response to COVID-19. Since many studies emphasized a significant amplified risk of COVID-19 infection among frontliners who are in direct contact with COVID-19 patients compared to the general community [27], there is an urgent need to improve the acceptance of COVID-19 vaccine particularly among this category.

The study's main findings are that nearly $58 \%$ of surveyed participants were willing to receive the COVID-19 vaccine. Our results were consistent with the findings displayed in a systematic review regarding acceptability of COVID-19 vaccine which found that the proportion of HCWs that intent to accept COVID-19 vaccination was $55.9 \%$ with a wide range among studies from $27.7 \%$ to $81.5 \%$. In United States of America (USA), a recent preprint research paper reports that vaccine acceptance is low even among HCWs [28]. This willingness to get vaccinated increased with age. Our results are concordant with those of a study conducted in DR Congo among HCWs [29]. An increase of acceptance of vaccine among older HCWs may be due to the fear expressed by elderly, given the serious complications and deaths recorded amongst them. Geographical variation in vaccine acceptance was also found in our survey. Peripheral areas such as Akkar province had the lowest acceptance rate comparing to the capital (Beirut). 
One noteworthy finding in the present study is that influenza vaccination coverage during the current season escalates to $67.3 \%$ comparing to the past season where only $45.4 \%$ of surveyed HCWs have received their influenza vaccine. This could be due to the fact that public health experts highlight the importance of influenza vaccine during the COVID-19 pandemic which overlapped with the influenza season. It is also indicating a generally promising perception of vaccination. However, only $18.94 \%$ of them had reported that they have previous experience of refusal of a type of vaccine other than influenza. This behavior defined as vaccine hesitancy could be related to confidence issues, complacency and convenience [30].

Concerning HCWs' perception of susceptibility, the majority of participants perceived themselves susceptible to get COVID-19 due to their occupational exposure. A risk perception assessment of COVID-19 among Portuguese HCWs showed that 54.9\% perceived themselves at a high likelihood of becoming infected [31]. Another study conducted in USA showed that HCWs, particularly nurses, have a higher prevalence of SARS-CoV-2 infection than non-health care workers, according to researchers at Rutgers [32].

Notably, $91.6 \%$ of HCWs considered that healthy people can get COVID-19 infection and could need hospitalization. These findings were in line with the outcomes of a study conducted among more than 3,000 adults aged between 18 and 34 years who contracted COVID-19 and required hospitalization. Researchers reported that $21 \%$ of them ended up in intensive care, $10 \%$ were placed on a breathing machine and $2.7 \%$ died [33] On the other hand, only half of them declared that they can protect themselves better than other people. One possible explanation could be that this proportion of HCWs consider themselves more aware and knowledgeable of the latest information of COVID-19 pandemic. Their skills, received training and their adherence to basic infection prevention measures such as basic hygiene (hand and cough etiquette), the availability and adequate use of protective personal equipment make them feeling safer and more protected against infectious diseases. Indeed, HCWs were mentioned as the 'canary in the mine'.

More than $90 \%$ of HCWs agreed that COVID-19 makes some people ill and could be fatal and $85.6 \%$ considered it more serious than influenza [34]. In this regard, many health experts highlighted the large numbers of susceptible people due to the slight preexistent immunity to the virus that causes COVID-19 infection which allows it to blowout so easily and increasing rates of severe disease and deaths.

Only $29.3 \%$ of them thought that they will be very ill if they contracted COVID-19, $30.7 \%$ stated that they may need hospitalization and $21.7 \%$ pondered that they might die. Our results were consistent with the findings of a study conducted in China, which revealed that the proportion of HCWs with severe illness was high in early January 2020 then decreased reflecting more steady adoption of IPC [35]. However, severe illness among HCWs continues to be reported, suggesting that the use of currently approved infection control processes do not entirely prevent severe COVID-19 among HCWs.

Our participants mentioned that the main benefits of getting vaccinated against COVID-19 include decreasing their fears about catching COVID19 , decreasing their chance of getting infected or presenting complications, protecting their patients and family members and preventing the spread of COVID-19 at community level. This comes in consistency with the findings of a study conducted among Chinese population who perceived the same benefits [36].

The main barriers cited by HCW's that could hamper their willingness to vaccinate against COVID-19 were their concerns about novelty, side effects, efficacy, and the vaccine safety. There are vigorous testing trials in place to warrant that approved COVID-19 vaccines are both effective and safe [37]. Bearing in mind that all kind of therapies, including vaccination, has risks. Addressing the extent of those risks presents a biggest challenge in the development of the COVID-19 vaccine.

In terms of gender, our results also revealed some interesting results, males were 2 times more likely to get vaccinated compared to males. Our results were consistent with other studies that showed that men were more willing to get vaccinated compared to women. This could be explained by the high infection rate of COVID-19 among them. A study conducted among Newzeelanders showed that two-thirds of women were willing to be vaccinated compared to three-quarters of men [38].

Besides, working in the frontline was associated positively to vaccine acceptance; this may be due to the highest risk of susceptibility faced by this category of HCWs. Our results were in line with a study conducted in USA that found that vaccine acceptance was higher among HCWs involved in direct patient care [39]. It is worth mentioning that past behavior handed over by receiving influenza vaccination in the current season was positively associated with the COVID-19 vaccine acceptance.

Having personal history of COVID-19 infection was negatively associated with the willingness to vaccinate, this could be due to the fact that the majority of previously infected people considered themselves as naturally immune, hence they refused to get vaccinated.

The majority of the HBM domains were considerably associated with the vaccine uptake. In particular, respondents who perceived the vaccine as conferring benefits, and received cues to action were significantly more likely to accept the vaccine. Our results were consistent with the outcomes of a study conducted in China [40].

Page $13 / 19$ 
Of note, we found that some keys components of HBM such us perceived susceptibility and perceived severity of infection were not associated with the vaccine acceptance. This observation could be explained by the fact that COVID-19 is perceived as a mild disease unless the infected person has underlying risk conditions. This implies the direction of promotion and education for COVID-19 vaccine should be different than other vaccines in which prevention of infection is perceived as the main purpose.

On the other hand, perceived barriers such as novelty of vaccine, possible side effects induced by the vaccine,reliability of manufacturer, trust of the source and number of doses required to get immune were negatively associated with the vaccine uptake. Our results were in line with the facts that trust in vaccination manufacturer and source contributes to explain vaccination uptake [41]. Given the massive plea of COVID 19 vaccine, a large number of new companies entered to the market [42]. However, the lack of awareness besides the large number of suppliers at once would intensify the doubt of vaccine recipients towards the validity of less recognized producers, which may in turn limit their acceptance of vaccines. In order to crack this obstacle, governments should also proactively include details about their chosen vaccine manufacturer(s). A peculiar finding that HCWs who conceived limited availability and accessibility of COVID-19 vaccine as barriers were more likely to accept vaccine. This could be explained by the facts that were usually the unreachable makes things unspeakably desirable. However, it is not universal human nature but a common learned pattern.

The main predictor of the willingness to vaccinate was the perception of benefits, HCWs who perceived the benefits were 10.771 more likely to get vaccinated than their counterparts with low perception of benefits. Our results were concordant with a study conducted in France that stresses the importance of communicating the benefits of herd immunity in reducing COVID-19 vaccine hesitancy [43].

With regards to cues of action, we found a remarkable pattern for COVID-19 vaccine. The recommendation from the health authorities and from the health facilities stood out as the most important cues. However, family members' recommendation that is associated negatively to COVID19 vaccine associated with acceptance. A substantial discrepancy was recorded by country, with China again having the highest proportion of positive responses (83.7\%) comparing to Russia that had the highest proportion of negative responses willing to accept their employer's recommendation [44].

Furthermore, having reliable, sufficient and adequate information regarding the vaccine also turn out to be an important driver of vaccine acceptance. These observations highlight evidence-based design of vaccine promotion campaigns tailored for the context of the HCWs concern.

Consistently to other studies that demonstrated the capability of the HBM constructs in predicting behaviors related to influenza vaccination [45]. Our findings suggested that the domains of HBM could be used to elucidate vaccine uptake behavior.

In summary, this study revealed a moderate acceptance rate of COVID-19 vaccine among participated HCWs and this could have broader extents. Our findings highlighted also the significance of governmental and facility recommendation on vaccine uptake, whereas knowledge, perceived susceptibility and severity to infection were not. Acceptance could be impaired by worries on novel vaccination approaches and manufacturers reliability. It will also guide us to develop the appropriate interventions to increase COVID-19 vaccination uptake.

There is a significant need for addressing concerns and increasing awareness to improve acceptance rate for COVID-19 vaccine. Otherwise, there is an additional chance of mass hesitancy among the general population when the COVID-19 vaccine becomes available.

\section{Limitations of the study:}

Several limitations of this study should be acknowledged. Firstly, this is a cross-sectional survey and we could not establish a cause-and-effect relationship between the independent factors and the outcome. They could however be used in prediction of COVID-19 vaccine acceptance. Also, this survey examined the HBM constructs among Lebanese HCWs, and the generalizability of its findings to other settings should be cautious. Secondly, our study relies on HCWs self-reported information, which may be a threat to information bias. In addition, self-reported information could be associated with recall bias about past behaviors. On other hand, participant's responses may be influenced by social desirability and then intentionally modified to meet the norms. Finally, since the online survey relies on the availability and accessibility to internet, there is a possibility of selection bias.

\section{Conclusion}

The moderate acceptance of HCWs to vaccinate against COVID-19 could impact the trust of individuals and trigger a ripple effect in the general public. Since vaccination is a complex behavior, understanding and pointing out the factors driving moderate vaccine uptake via targeting messages addressing key concerns would be required to reach higher vaccination rate.

\section{Declarations}




\section{Ethics approval}

The study design respected the participant's confidentiality and autonomy. A written informed consent was obtained from all participants. As survey studies are exempt from ethical approval in Lebanon according to national regulations [46], the Lebanese Ministry of Public Health waived the need for ethical approval.

\section{Funding}

This research did not receive any specific grant from funding agencies in the public, commercial, or not-for-profit sectors.

\section{Data availability}

After publication, the survey data will be made available on reasonable request to the corresponding author. A proposal with a detailed description of study objectives and a statistical analysis plan will be needed for assessment of requests. Additional materials might also be required during the process of assessment. De-identified participant data will be provided after approval by the investigators.

\section{Authors' contributions}

DY developed the project idea. DY, JY and LAA formulated the questionnaire, organized and analyzed the survey. DY, JY, LAA, AB and HS drafted and critically reviewed the paper. All authors read and agreed on the final version.

\section{Declaration of Competing Interest}

The authors declare that they have no known competing financial interests or personal relationships that could have appeared to influence the work reported in this paper.

\section{Transparency declaration}

The corresponding author, as the manuscript's guarantor, affirms that the manuscript is an honest, accurate, and transparent account of the study being reported and that no important aspects of the study have been omitted.

\section{Consent for publication}

Not applicable.

\section{Acknowledgments}

We would like to acknowledge all health care workers participating in the study

\section{Authors information}

Dalal Youssef, MSc, MPH, PHD candidate, Ministry of public Health, Lebanon, Preventive medicine department, Lebanon

Janet Youssef, MD, OBGYN, ZHUMC, Lebanon

Atika Berry, MD, Ministry of Public Health, Preventive medicine department, Lebanon

Linda Abou Abbas, MPH, PHD, Ministry of Public Health, Epidemiological surveillance unit, Lebanon

Hamad Hassan, PhD, Ministry of Public Health, Lebanon

\section{References}


1. Haque, M., The COVID-19 Pandemic - A Global Public Health Crisis: A Brief Overview Regarding Pharmacological Interventions. Pesquisa Brasileira em Odontopediatria e Clínica Integrada, 2020. 20.

2. COVID-19 Dashboard by the Center for Systems Science and Engineering (CSSE) at Johns Hopkins University. Available: https://coronavirus.jhu.edu/map.html. Accessible: 23/02/2021

3. Nicola, M., et al., Evidence based management guideline for the COVID-19 pandemic - Review article. International Journal of Surgery, 2020. 77: p. 206-216.

4. Bubar, K.M., et al., Model-informed COVID-19 vaccine prioritization strategies by age and serostatus. Science, 2021.

5. Alturki, S.O., et al., The 2020 Pandemic: Current SARS-CoV-2 Vaccine Development. Front Immunol, 2020. 11: p. 1880.

6. Kaur, S.P. and V. Gupta, COVID-19 Vaccine: A comprehensive status report. Virus Research, 2020. 288: p. 198114.

7. PFIZER AND BIONTECH ACHIEVE FIRST AUTHORIZATION IN THE WORLD FOR A VACCINE TO COMBAT COVID-19. Available athttps://www.pfizer.com/news/press-release/press-release-detail/pfizer-and-biontech-achieve-first-authorization-world.Accessed 23/2/2021.

8. Forni, G., et al., COVID-19 vaccines: where we stand and challenges ahead. Cell Death \& Differentiation, 2021. 28(2): p. 626-639.

9. Wouters, O.J., et al., Challenges in ensuring global access to COVID-19 vaccines: production, affordability, allocation, and deployment. The Lancet, 2021.

10. Buckner, J.H., G. Chowell, and M.R. Springborn, Dynamic Prioritization of COVID-19 Vaccines When Social Distancing is Limited for Essential Workers. medRxiv, 2020: p. 2020.09.22.20199174.

11. Levin, A.T., et al., Assessing the age specificity of infection fatality rates for COVID-19: systematic review, meta-analysis, and public policy implications. European Journal of Epidemiology, 2020. 35(12): p. 1123-1138.

12. Yang, J., et al., Who should be prioritized for COVID-19 vaccination in China? A descriptive study. BMC Medicine, 2021. 19(1): p. 45.

13. World Health Organization (WHO). Keep health workers safe to keep patients safe: WHO. Available athttps://www.who.int/news/item/1709-2020-keep-health-workers-safe-to-keep-patients-safe-who.Accessed on 23/2/2021.

14. World Health Organization. WHO SAGE Roadmap For Prioritizing Uses Of COVID-19 Vaccines In The Context Of Limited Supply. Available at. https://www.who.int/publications/m/item/who-sage-roadmap-for-prioritizing-uses-of-covid-19-vaccines-in-the-context-of-limitedsupply.Accessed 23/2/2021.

15. Countdown to a COVID-19 Vaccine: Spotlight on Lebanon. Available at. https://www.aub.edu.lb/k2p/Documents/K2P\%20COVID19\%20Series_Countdown\%20to\%20a\%20COVID19\%20Vaccine.pdf. Accessed on $23 / 2 / 2021$.

16. Ministry of Public Health. Novel Coronavirus 2019. COVID-19 surveillance in Lebanon. Available at. https://www.moph.gov.lb/en/Pages/2/24870/novel-coronavirus-2019-. Accessed on 3/3/2021.

17. Lebanon National Deployment and Vaccination Plan for COVID-19 Vaccines. Available at. https://www.moph.gov.lb/userfiles/files/Prevention/COVID-19\%20Vaccine/Lebanon\%20NDVP-\%20Feb\%2016\%202021.pdf. Accessed on 29/1/2021

18. Alhalaseh, L., H. Fayoumi, and B. Khalil, The Health Belief Model in predicting healthcare workers' intention for influenza vaccine uptake in Jordan. Vaccine, 2020. 38(46): p. 7372-7378.

19. Jones, C.L., et al., The Health Belief Model as an explanatory framework in communication research: exploring parallel, serial, and moderated mediation. Health communication, 2015. 30(6): p. 566-576.

20. Beaton, D., et al., Guidelines for the Process of Cross-Cultural Adaption of Self-Report Measures. Spine, 2001. 25: p. $3186-91$.

21. Myers, L.B. and R. Goodwin, Determinants of adults' intention to vaccinate against pandemic swine flu. BMC Public Health, 2011. 11(1): p. 15.

22. Coe, A.B., et al., The use of the health belief model to assess predictors of intent to receive the novel (2009) H1N1 influenza vaccine. Innov Pharm, 2012. 3(2): p. 1-11.

23. Guidry, J.P.D., et al., Willingness to get the COVID-19 vaccine with and without emergency use authorization. American Journal of Infection Control, 2021. 49(2): p. 137-142.

24. COVID-19: Guidance for Prioritizing Health Care Workers for COVID-19 Vaccination. Available at. http://www.health.gov.on.ca/en/pro/programs/publichealth/coronavirus/docs/Guidance_for_Prioritizing_HCW_covid19_vaccination_202001-08.pdf. Accessed 20/2/2021.

25. Alajmi, J., et al., COVID-19 infection among healthcare workers in a national healthcare system: The Qatar experience. International journal of infectious diseases: IJID : official publication of the International Society for Infectious Diseases, 2020. 100: p. 386-389. 
26. Mahajan, N.N., et al., Prevalence and Clinical Presentation of COVID-19 among Healthcare Workers at a Dedicated Hospital in India. J Assoc Physicians India, 2020. 68(12): p. 16-21.

27. Nguyen, L.H., et al., Risk of COVID-19 among frontline healthcare workers and the general community: a prospective cohort study. medRxiv: the preprint server for health sciences, 2020: p. 2020.04.29.20084111.

28. Galanis, P.A., et al., Intention of health care workers to accept COVID-19 vaccination and related factors: a systematic review and metaanalysis. medRxiv, 2020: p. 2020.12.08.20246041.

29. Kabamba Nzaji, M., et al., Acceptability of Vaccination Against COVID-19 Among Healthcare Workers in the Democratic Republic of the Congo. Pragmatic and observational research, 2020. 11: p. 103-109.

30. MacDonald, N.E., Vaccine hesitancy: Definition, scope and determinants. Vaccine, 2015. 33(34): p. 4161-4164.

31. Peres, D., et al., Risk Perception of COVID-19 Among the Portuguese Healthcare Professionals and General Population. The Journal of hospital infection, 2020. 105(3): p. 434-437.

32. Barrett, E.S., et al., Prevalence of SARS-CoV-2 infection in previously undiagnosed health care workers in New Jersey, at the onset of the U.S. COVID-19 pandemic. BMC Infectious Diseases, 2020. 20(1): p. 853.

33. Cunningham, J.W., et al., Clinical Outcomes in Young US Adults Hospitalized With COVID-19. JAMA Internal Medicine, 2020.

34. Piroth, L., et al., Comparison of the characteristics, morbidity, and mortality of COVID-19 and seasonal influenza: a nationwide, populationbased retrospective cohort study. The Lancet Respiratory Medicine, 2020.

35. Lai, X., et al., Coronavirus Disease 2019 (COVID-2019) Infection Among Health Care Workers and Implications for Prevention Measures in a Tertiary Hospital in Wuhan, China. JAMA Network Open, 2020. 3(5): p. e209666-e209666.

36. Lin, Y., et al., Understanding COVID-19 vaccine demand and hesitancy: A nationwide online survey in China. PLoS Negl Trop Dis, 2020. 14(12): p. e0008961.

37. World Health Organization. An international randomised trial of candidate vaccines against COVID-19. [accessed 2020 May 26].https://www.who.int/publications-detail/an-international-randomised-trial-of-candidate-vaccines-against-covid-19.

38. Research New Zealand. New Zealanders Have Some Concerns Around COVID-19 Vaccinations. Available athttps://www.scoop.co.nz/stories/GE2102/S00059/new-zealanders-have-some-concerns-around-covid-19-vaccinations.htm.Accessed 25/2/2021.

39. Shekhar, R., et al., COVID-19 Vaccine Acceptance among Health Care Workers in the United States. Vaccines (Basel), 2021. 9(2).

40. Wong, M.C.S., et al., Acceptance of the COVID-19 vaccine based on the health belief model: A population-based survey in Hong Kong. Vaccine, 2021. 39(7): p. 1148-1156.

41. Larson, H.J., et al., Measuring trust in vaccination: A systematic review. Human vaccines \& immunotherapeutics, 2018. 14(7): p. 15991609.

42. Ned P, Gardner J, Fidler B. The coronavirus vaccine frontrunners are advancing quickly. Here's where they stand. / BioPharma dive. Retrieved September 18, 2020 Available at. https://www.biopharmadive.com/news/coronavirus-vaccine-pipeline-types/579122/ [accessed on 20 September 2020].

43. Schwarzinger, M., et al., COVID-19 vaccine hesitancy in a representative working-age population in France: a survey experiment based on vaccine characteristics. The Lancet Public Health, 2021.

44. Lazarus, J.V., et al., A global survey of potential acceptance of a COVID-19 vaccine. Nature Medicine, 2021. 27(2): p. 225-228.

45. Mo, P.K. and J.T. Lau, Influenza vaccination uptake and associated factors among elderly population in Hong Kong: the application of the Health Belief Model. Health Educ Res, 2015. 30(5): p. 706-18.

46. Ministry of Public Health, Standards and Operational Guidance for Ethics Review of Health-Related Research with Human Participants, https://www.moph.gov.lb/userfiles/files/HealthCareSystem/Pharmaceuticals/ClinicalTrial/WHOGuidelines.pdf, accessed February 1, 2021.

\section{Figures}


HCW' willingness to receive COVID-19 vaccine when the vaccine is available in Lebanon $(\mathrm{N}=1800)$

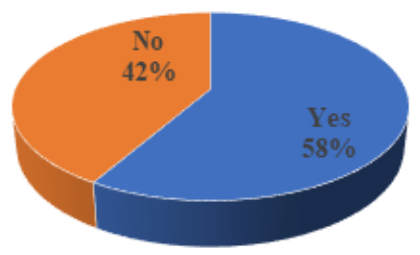

\section{Figure 1}

HCWs' willingness to receive COVID-19 vaccine when the vaccine is available in Lebanon ( $\mathrm{N}=1800)$

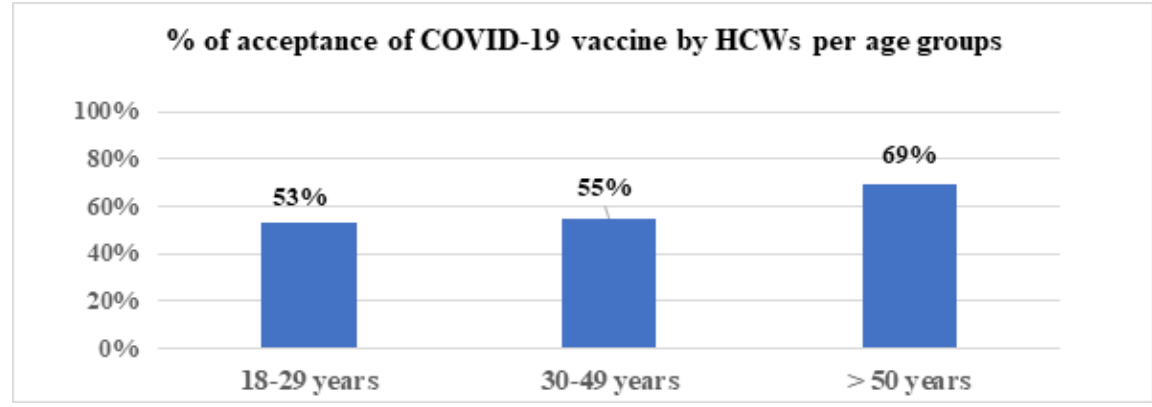

\section{Figure 2}

HCWs' intention to receive COVID-19 vaccine per age groups

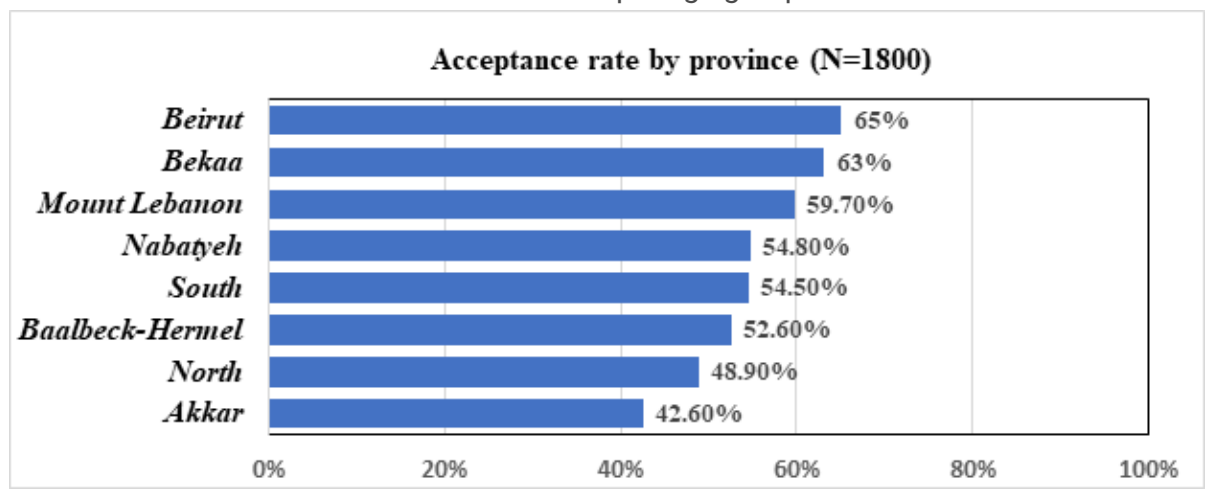

\section{Figure 3}

HCWs' intention to receive COVID-19 vaccine per age groups

\section{Knowledge score}

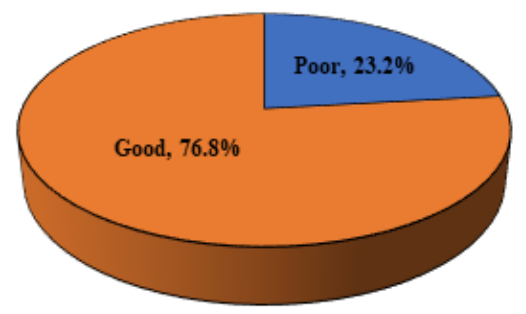

Figure 4

knowledge score 


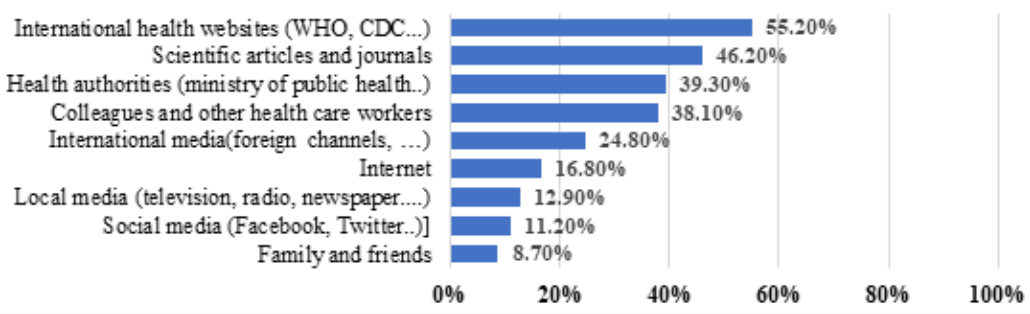

\section{Figure 5}

HCWs' ranking of the most reliable source of information

\section{Supplementary Files}

This is a list of supplementary files associated with this preprint. Click to download.

- QuestionnaireDeterminantsofacceptanceofCOVID.docx 